\title{
The concordance of directly and indirectly measured built environment attributes and physical activity adoption
}

\author{
Kristen M McAlexander ${ }^{1,2^{*}}{ }$, Scherezade K Mama ${ }^{2 \dagger}$, Ashley Medina ${ }^{2 \dagger}$, Daniel P O'Connor ${ }^{2 \dagger}$ and Rebecca E Lee $^{2 \dagger}$
}

Background: Physical activity (PA) adoption is essential for obesity prevention and control, yet ethnic minority women report lower levels of PA and are at higher risk for obesity and its comorbidities compared to Caucasians. Epidemiological studies and ecologic models of health behavior suggest that built environmental factors are associated with health behaviors like PA, but few studies have examined the association between built environment attribute concordance and PA, and no known studies have examined attribute concordance and PA adoption.

Purpose: The purpose of this study was to associate the degree of concordance between directly and indirectly measured built environment attributes with changes in PA over time among African American and Hispanic Latina women participating in a PA intervention.

Method: Women $(N=410)$ completed measures of PA at Time 1 (T1) and Time 2 (T2); environmental data collected at T1 were used to compute concordance between directly and indirectly measured built environment attributes. The association between changes in PA and the degree of concordance between each directly and indirectly measured environmental attribute was assessed using repeated measures analyses.

Results: There were no significant associations between built environment attribute concordance values and change in self-reported or objectively measured PA. Self-reported PA significantly increased over time $(F(1,184)=$ $7.82, p=.006)$, but this increase did not vary by ethnicity or any built environment attribute concordance variable.

Conclusions: Built environment attribute concordance may not be associated with PA changes over time among minority women. In an effort to promote PA, investigators should clarify specific built environment attributes that are important for PA adoption and whether accurate perceptions of these attributes are necessary, particularly among the vulnerable population of minority women.

\section{Background}

Ethnic minority women report lower levels of physical activity (PA) [1] and are at higher risk for obesity and its comorbidities compared to Caucasian women $[2,3]$. Further, health attitudes and behaviors can differ by ethnicity [4-6]. Studies that investigate built environment measurement factors related to the adoption of PA are extremely important since consistent evidence suggests that neighborhood characteristics and health behaviors

\footnotetext{
* Correspondence: kmcalexander@smu.edu

† Contributed equally

'Department of Applied Physiology and Wellness, Southern Methodist University, Dallas, TX, USA

Full list of author information is available at the end of the article
}

are significantly related [7-10]. Research suggests that factors influencing PA adoption are different for men and women $[11,12]$, and there may be different factors influencing behavior adoption versus maintenance $[13,14]$.

Ecologic models of human behavior have evolved over decades in the fields of sociology, psychology and public health [7,15-17], and their significance to PA is now widely recognized [7,16-18]. Neighborhood built environment changes can benefit all people in a surrounding neighborhood rather than only focusing on changing individual behavior [17]. These changes can include building and improving physical activity resources (PARs), sidewalks

\section{Biomed Central}


and bicycle facilities (e.g., bicycle lanes, bicycle route signs), and can be more permanent than interventions focusing on individual-level changes. Specific built environment attributes can provide opportunities, support, and cues to help people adopt PA and may complement individual-level programs. Empirical evidence consistently supports these associations [7-10], but less is known about how built environment attributes affect PA adoption, especially among vulnerable populations like women [17] for whom predictors of the adoption and maintenance of PA can differ [19].

The concordance of directly measured built environment attributes and indirectly measured built environment attributes has been significantly associated with PA $[20,21]$. Concordance is measured by the strength and direction of the correlation between directly measured and indirectly measured variables of the built environment [20,22]. Direct built environment measures may provide objective data, unbiased by resident perceptions, as well as specific evidence for policy change impacting urban planning and transportation. Indirect built environment measures include self-reported data on perceived environmental attributes and can provide insight on individual attitudes about the built environment. Both direct and indirect measures of the built environment have been associated with PA separately [10,23-26], but few studies have examined the association between concordance and PA [20,21,27]. Gebel and colleagues found a fair overall agreement between objectively determined walkability and perceived walkability, but adults with lower educational attainment and lower incomes or who were overweight were more likely to misperceive their high walkable neighborhood as low walkable [20]. Findings suggest the potential for PA promotion and persuasion strategies to address non-concordance [20], but these associations have not been examined for PA adoption or among minority women. Individuals who are less physically active may also be more likely to misperceive their built environment compared to those who are more physically active $[20,27]$, suggesting that the concordance of direct and indirect built environment measurement may be dynamic and related to PA and/or PA adoption.

The purpose of this study was to measure the associations between built environment attribute concordance and PA adoption among African American and Hispanic or Latina women. We hypothesized that women who demonstrated a stronger concordance between directly and indirectly measured built environment attributes would exhibit increased PA over time or PA adoption.

\section{Methods}

The current study is a secondary analysis using data from the Health is Power (HIP) project. Originating in 2005, the HIP project was a five-year, longitudinal study funded by the National Cancer Institute of the National Institutes of Health (R01 CA109403) to increase PA and improve dietary habits in African American and Hispanic or Latina women in Houston and Austin, Texas. The HIP project was approved by the Committee for the Protection of Human Subjects at the University of Houston, and participants provided written informed consent to participate. The investigators certified that all applicable institutional and governmental regulations concerning the ethical use of human research volunteers were followed during the investigation.

\section{Study Design}

Environmental cross-sectional data and longitudinal individual data were used to measure the association between concordance between directly and indirectly measured built environment attribute data and changes in PA over time among African American and Hispanic or Latina women.

\section{Participants}

Four hundred ten African American and Hispanic or Latina women (311 in Houston and 99 in Austin) were enrolled in the study. Of those enrolled in Houston, 84.6\% identified as African American and 15.4\% identified as Hispanic or Latina; all participants in Austin identified as Hispanic or Latina [28].

\section{Measures}

\section{Individual Measures}

Sociodemographic measures of age, gender, marital status, employment status, years of education, and income range were measured using the Maternal and Infant Health Assessment (MIHA) [29]. Modeled on the Center for Disease Control's (CDC) Pregnancy Risk Assessment Monitoring System (PRAMS), the MIHA includes items that have been used with samples representing a diverse range of ethnicities and socioeconomic status categories $[29,30]$.

To assess self-reported PA levels, the International Physical Activity Questionnaire (IPAQ) Long Form was used. Median values and interquartile ranges were computed for walking, moderate-intensity activities, vigorous-intensity activities and for a combined total PA score. The total PA score at Time 1 (T1) was used along with the total PA score at Time 2 (T2) to measure changes, or differences, in PA from T1 to T2. All continuous scores were expressed in MET-minutes, computed by multiplying the MET score of an activity by the minutes performed [31].

Accelerometers (MTI Actigraph) were used to objectively assess the amount and intensity of PA participants did each day [32]. Participants wore accelerometers for seven consecutive days at to assess typical PA for moderate-intensity or greater activity. Days with eight or 
more valid hours of data, or fewer than 30 consecutive zero counts, were included in analyses [32]. A daily average of the amount of moderate-vigorous accelerometer-measured PA (MVPA) at T1 and T2 was used to measure changes in $\mathrm{PA}$ from $\mathrm{T} 1$ to $\mathrm{T} 2$, or $\mathrm{PA}$ adoption.

Body Mass Index $\left(B M I=\mathrm{kg} / \mathrm{m}^{2}\right)$ and percent body fat were used as measures of body composition. Participants removed shoes and heavy outer clothing, and trained research assistants measured height using a portable stadiometer (Seca 225 Hite Mobile Measuring Device; North Bend, Washington) and weight using a bioelectrical impedance monitor with scales (The TBF310 \& the TBF-300; Tanita Corporation, Chicago of America, Arlington Heights, IL). Percent body fat was measured using the Tanita integrated bioelectrical impedance body fat monitor and scale (Tanita Body Fat Analyzer, TBF 105, Tanita Corporation of America, Inc., Arlington Heights, IL).

\section{Procedures}

\section{Individual Assessments}

Women were recruited to the HIP project via the media, brochures, churches and internet communication over the course of one year. Interested participants were invited to call the HIP project and complete a telephone screener. Women were screened to meet the following inclusion criteria: (1) self identified as African American or Hispanic or Latina, (2) between the ages of 25 and 60 years old, to include adults outside the college age range, (3) able to read, speak, and write in English or Spanish, (4) not pregnant or planning to become pregnant within the next 12 months, (5) a Harris or Travis County resident, (6) not planning on moving in the next 12 months, (7) physically inactive or doing fewer than 30 minutes of physical activity per day on 3 or more days per week, and (8) able to pass the Physical Activity Readiness Questionnaire (PAR-Q) [33]. Eligible participants completed an interviewer administered self-report environmental perception questionnaire at T1 and self-reported PA measures at T1 and T2. Participants also completed a seven day accelerometer protocol at $\mathrm{T} 1$ and $\mathrm{T} 2$ and were compensated for completing assessments at each time point [32].

\section{Neighborhood Assessments and GIS Development}

As reported previously [34], participant street addresses were geocoded and plotted by a trained Geographical Information Systems (GIS) specialist using ArcGIS software [35]. Each participant's neighborhood was restricted to an 800 meter or approximately $1 / 2$ mile radius buffer. Environmental assessments were completed during the intervention to capture neighborhoods at the same time in order to avoid simultaneity bias [36]. In order to compare directly measured PAR accessibility to indirectly measured PAR accessibility, the total number of accessible PARs was calculated for each participant's neighbourhood using the Physical Activity Resource Assessment instrument [37-39]. Path maintenance was assessed based on the amount of debris and/or the overall condition of the facility, and pedestrian and bicycle facility density was calculated by counting the number of pedestrian and bicycle facilities within each predefined neighborhood (i.e. $800 \mathrm{~m}$ radius circle) using the Pedestrian Environment Data Scan instrument $[35,40]$.

\section{Statistical Analyses}

Descriptive analyses were completed to examine the frequency and distribution of individual and environmental variables. BMI, body fat percentage, self-reported PA and accelerometry were analyzed at $\mathrm{T} 1$ and $\mathrm{T} 2$, and bivariate analyses were conducted among all variables, including directly measured and indirectly measured built environment variables, BMI, body fat percentage, self-reported PA, accelerometer measured PA, sociodemographic variables and ethnicity.

Repeated measures analyses were conducted to determine if concordance values were associated with PA adoption or PA changes from $\mathrm{T} 1$ to $\mathrm{T} 2$, for both the IPAQ and accelerometer measured PA. Because bivariate and model-based analyses suggested no significant associations among any individual and built environment variables, only ethnicity was included in the repeated measures analyses in order to examine differences among African Americans and Hispanic or Latinas. Interaction terms were considered in the models, and the F-ratio test significance was set at $p<.05$. All statistical analyses were conducted in SPSS Version 18.0 (SPSS 18.0 for Windows; SPSS Inc, Chicago, Ill).

\section{Results}

\section{Descriptive Characteristics}

Participants $(N=410)$ were mostly obese $(\mathrm{T} 1 M \mathrm{BMI}=$ $34.5 \mathrm{~kg} / \mathrm{m}^{2}, S D=7.9 ;$ T2 $M$ BMI $=34.2 \mathrm{~kg} / \mathrm{m}^{2}, S D=$ $8.1)$, highly educated (89\% completed college or completed some college) and nearly half reported an income over $400 \%$ of the Federal Poverty Level for a family of four in 2007 [41]. African American women $(M=$ 3326.5 MET minutes per week, $S D=3169.5$ and $M=$ 24.4 minutes MVPA per day, $S D=19.9)$ were more physically active than Hispanic or Latina women $(M=$ 2840.5 MET minutes per week, $S D=2067.0$ and $M=$ 11.7 minutes MVPA per day, $S D=9.1$ ) according to self-reported and objectively-measured PA assessments [28]. Ethnicity, BMI, percent body fat and PA were not significantly associated with any built environment attribute. All descriptive individual and environmental data have been reported previously $[28,34]$. 


\section{Built Environment Attribute Concordance and PA Adoption}

Repeated measures analyses revealed no significant relationships between any built environment attribute concordance value and PA adoption or PA changes from T1 to T2 for total self-reported or objectively measured PA. Self-reported PA significantly increased over time $(F(1,184)=7.82, p=.006)$ [28] but did not significantly vary by ethnicity, BMI, percent body fat and directly or indirectly measured built environment attributes. Objectively measured PA did not significantly increase over time [28]. Repeated measures analyses results are presented in Tables 1 and 2.

\section{Discussion}

We hypothesized that for our sample of minority women, a stronger concordance of directly and indirectly measured built environment attributes would be significantly associated with PA adoption. Objectively measured PA did not significantly increase, but selfreported PA did significantly increase from T1 to T2. PA changes over time did not vary by ethnicity or any concordance measure.

No earlier study has measured the association between built environment attribute concordance and PA changes over time, but PA has been reported to be a significant correlate of built environment attribute concordance [20]. In particular, one study found lower concordance among women with lower income, PA and self-efficacy for PA [27]. Also, other findings suggest that indirectly measured neighborhood data are more closely linked to self-reported PA than directly measured neighborhood data $[27,42]$. Unlike studies measuring direct and indirect built environment attribute concordance, our sample consisted solely of minority women. The relationships between PA and attribute concordance might differ for our population, as earlier findings suggest that the degree of built environment non-concordance can vary among certain population subgroups [27]. Also, our samples were of high SES, particularly for income and education; we also assessed a wider variety of neighborhood types than previous studies $[20,42]$, increasing the generalizability of our findings.

Although not all of our participants exhibited increased PA over time or PA adoption, this study initiates an evidence base where no similar data exist. PA adoption is an essential component to a healthy lifestyle $[3,43]$, yet no known study has measured the associations of PA changes over time with built environment concordance values. Further, this study investigated these relationships among minority women. Although African American and Hispanic or Latina women continue to be disproportionately

Table 1 Repeated Measures results for self-reported PA adoption

\begin{tabular}{|c|c|c|c|c|}
\hline Built Environment Concordance Attribute Used & Effect & $d f$ & $F$ & $p$-value \\
\hline \multicolumn{5}{|l|}{ PAR Access } \\
\hline & Time & 1 & 2.69 & .10 \\
\hline & Time*Ethnicity & 1 & .00 & .99 \\
\hline & Time*PAR Access Concordance & 1 & .12 & .73 \\
\hline & Time*Ethnicity*PAR Access Concordance & 1 & .71 & .40 \\
\hline & Error & 162 & & \\
\hline \multicolumn{5}{|l|}{ Path Maintenance } \\
\hline & Time & 1 & 2.39 & .12 \\
\hline & Time*Ethnicity & 1 & .01 & .91 \\
\hline & Time*Path Maintenance Concordance & 1 & .84 & .36 \\
\hline & Time*Ethnicity*Path Maintenance Concordance & 1 & .01 & .91 \\
\hline & Error & 153 & & \\
\hline \multicolumn{5}{|l|}{ Pedestrian Facility Density } \\
\hline & Time & 1 & 2.17 & .14 \\
\hline & Time*Ethnicity & 1 & .21 & .65 \\
\hline & Time*Pedestrian Facility Density Concordance & 1 & .16 & .69 \\
\hline & Time*Ethnicity*Pedestrian Facility Density Concordance & 1 & .47 & .49 \\
\hline & Error & 158 & & \\
\hline \multicolumn{5}{|l|}{ Bicycle Facility Density } \\
\hline & Time & 1 & 7.22 & .01 \\
\hline & Time*Ethnicity & 1 & .61 & .44 \\
\hline & Time*Bicycle Facility Density Concordance & 1 & .79 & .38 \\
\hline & Time*Ethnicity*Bicycle Facility Density Concordance & 1 & .44 & .51 \\
\hline & Error & 173 & & \\
\hline
\end{tabular}


Table 2 Repeated measures results for objectively-measured PA adoption

\begin{tabular}{|c|c|c|c|c|}
\hline Built Environment Concordance Attribute Used & Effect & $d f$ & $F$ & $p$-value \\
\hline \multicolumn{5}{|l|}{ PAR Access } \\
\hline & Time & 1 & 1.85 & .18 \\
\hline & Time*Ethnicity & 1 & .61 & .44 \\
\hline & Time*PAR Access Concordance & 1 & .20 & .66 \\
\hline & Time*Ethnicity*PAR Access Concordance & 1 & 1.83 & .19 \\
\hline & Error & 36 & & \\
\hline \multicolumn{5}{|l|}{ Path Maintenance } \\
\hline & Time & 1 & .80 & .38 \\
\hline & Time*Ethnicity & 1 & .29 & .59 \\
\hline & Time*Path Maintenance Concordance & 1 & .35 & .56 \\
\hline & Time*Ethnicity*Path Maintenance Concordance & 1 & .22 & .64 \\
\hline & Error & 35 & & \\
\hline \multicolumn{5}{|l|}{ Pedestrian Facility Density } \\
\hline & Time & 1 & 1.63 & .21 \\
\hline & Time*Ethnicity & 1 & .60 & .45 \\
\hline & Time*Pedestrian Facility Density Concordance & 1 & .86 & \\
\hline & Time*Ethnicity*Pedestrian Facility Density Concordance & 1 & .94 & .36 \\
\hline & Error & 36 & & \\
\hline \multicolumn{5}{|l|}{ Bicycle Facility Density } \\
\hline & Time & 1 & .118 & .73 \\
\hline & Time*Ethnicity & 1 & .07 & .80 \\
\hline & Time*Bicycle Facility Density Concordance & 1 & .23 & .63 \\
\hline & Time*Ethnicity*Bicycle Facility Density Concordance & 1 & .09 & .77 \\
\hline & Error & 40 & & \\
\hline
\end{tabular}

physically inactive compared to white women [2,3], they continue to be understudied in the built environment literature [17].

Other strengths of this study include the use of a selfreported PA questionnaire and accelerometry to measure PA changes over time, providing a comprehensive assessment of PA. Although similar studies have been cross-sectional in nature $[20,27,44]$, our study measured PA longitudinally. We also used measured BMI and body fat percentage, rather than self-report, helping to reduce bias and measurement error.

Our study is not without limitations. Due to adherence, cost and logistic reasons, the number of participants who wore accelerometers was lower than those who completed the self-reported PA questionnaire at T1 and T2. Resources are needed for future studies to recruit and assess an equal number of participants for multiple PA measures to provide a more comprehensive PA assessment. McCormack and colleagues found that residents' perceived behavior control cognitions were mediators in the relationship between the built environment and PA [45], and future work is needed to include additional individual-level variables that might help explain the variability of attribute perception(s) and PA changes among these populations.
This study investigated built environment measurement concordance and PA changes over time among minority women. Inaccurate perceptions of built environment attributes were not associated with PA level change. Future PA interventions and supportive communities could promote built environment attributes (e. g., park amenities, clean baseball fields, long walking trails) in an attempt to increase PA. Policies could attempt to increase facility and street signage in an effort to promote PA, particularly among ethnically diverse neighborhoods.

\section{Conclusions}

Although the influence of the built environment on individual health behaviors has been well established, more study of the interactions between specific built environment attributes and intra-individual factors like gender and ethnicity is needed. These linkages are not well understood, and the applicability of ecological frameworks could be limited if the relationships between built environment attributes and health behaviors vary for certain personal characteristics. In an effort to promote PA, investigators should clarify specific built environment attributes that are important for PA adoption and whether accurate perceptions of these attributes are 
necessary, particularly among the vulnerable population of minority women.

\section{List of abbreviations}

BMI: Body Mass Index; CDC: Center for Disease Control and Prevention; GIS: Geographical Information Systems; HIP: Health is Power; IPAQ: International Physical Activity Questionnaire; MIHA: Maternal and Infant Health Assessment; MVPA: Moderate and Vigorous Physical Activity; PA: Physical Activity; PAR: Physical Activity Resource; PARA: Physical Activity Resource Assessment Instrument; PAR-Q: Physical Activity Readiness Questionnaire; PEDS: Pedestrian Environment Data Scan; PRAMS: Pregnancy Risk Assessment Monitoring System; SES: Socioeconomic Status; T1: Time 1; T2: Time 2.

\section{Acknowledgements}

1. Health Is Power (HIP) was a five-year, longitudinal study funded by the National Cancer Institute of the National Institutes of Health (R01 CA109403) awarded to Dr. Lee.

\section{Author details}

'Department of Applied Physiology and Wellness, Southern Methodist University, Dallas, TX, USA. ${ }^{2}$ Texas Obesity Research Center, Department of Health and Human Performance, University of Houston, Houston, TX, USA.

\section{Authors' contributions}

KMM primarily wrote the manuscript. SKM helped to coordinate the study and assisted with data collection. AM provided geographic data support and also helped with data collection. DPO assisted with analyses and interpretation of data. REL conceived the original study, secured funding, provided individual and environmental data and intensive guidance through all phases of the manuscript. All authors read and approved the final manuscript.

\section{Competing interests}

The authors declare that they have no competing interests.

Received: 17 September 2010 Accepted: 7 July 2011

Published: 7 July 2011

\section{References}

1. Kruger J, Yore MM, Kohl HW: Physical activity levels and weight control status by body mass index, among adults - National Health and Nutrition Examination Survey 1999-2004. Int J Behav Nutr Phys Act 2008, 5:25.

2. Ogden CL, Carroll MD, Curtin LR, McDowell MA, Tabak CJ, Flegal KM: Prevalence of overweight and obesity in the United States, 1999-2004. JAMA 2006, 295:1549-1555.

3. A Report of the Surgeon General. Physical Activity and Health 1996, 1-4.

4. Gordon-Larsen P, McMurray RG, Popkin BM: Adolescent physical activity and inactivity vary by ethnicity: The National Longitudinal Study of Adolescent Health. J Pediatr 1999, 135:301-306.

5. Harris MB, Walters LC, Waschull S: Gender and ethnic differences in obesity related behaviors and attitudes in a college sample. J App/ SoC Psychol 1991, 21:1545-1566.

6. Stern MP, Pugh JA, Gaskill SP, Hazuda HP: Knowledge, attitudes, and behavior related to obesity and dieting in Mexican Americans and Anglos: the San Antonio Heart Study. Am J Epidemiol 1982, 115:917-928.

7. Sallis JF, Bauman A, Pratt M: Environmental and policy interventions to promote physical activity. Am J Prev Med 1998, 15:379-397.

8. Owen N, Humpel N, Leslie E, Bauman A, Sallis JF: Understanding environmental influences on walking; Review and research agenda. Am J Prev Med 2004, 27:67-76.

9. Lee RE, Cubbin C, Winkleby M: Contribution of neighbourhood socioeconomic status and physical activity resources to physical activity among women. J Epidemiol Community Health 2007, 61:882-890.

10. Heinrich KM, Lee RE, Suminski RR, Regan GR, Reese-Smith JY, Howard HH, Haddock CK, Poston WS, Ahluwalia JS: Associations between the built environment and physical activity in public housing residents. Int J Behav Nutr Phys Act 2007, 4:56.
11. Biddle S, Bailey C: Motives toward participation and attitudes toward physical activity of adult participants in fitness programs. Percept Mot Skills 1985, 61:831-834.

12. Caspersen CJ, Merritt RK, Health GW, Yeager KK: Physical activity patterns of adults aged 60 years and older. Med Sci Sports Exerc 1990, 22(Suppl): S79.

13. Dunton GF, Vaughan E: Anticipated affective consequences of physical activity adoption and maintenance. Health Psychol 2008, 27:703-710.

14. Marshall SJ, Biddle SJ: The transtheoretical model of behavior change: a meta-analysis of applications to physical activity and exercise. Ann Behav Med 2001, 23:229-246.

15. Green LW, Richard L, Potvin L: Ecological foundations of health promotion. Am J Health Promot 1996, 10:270-281.

16. Spence J, Lee R: Toward a comprehensive model of physical activity. Psychology of Sport and Exercise 2003, 4:7-24.

17. Lee RE, Cubbin C: Striding toward social justice: the ecologic milieu of physical activity. Exerc Sport Sci Rev 2009, 37:10-17.

18. McLeroy KR, Bibeau D, Steckler A, Glanz K: An ecological perspective on health promotion programs. Health Educ Q 1988, 15:351-377.

19. Sallis JF, Hovell MF, Hofstetter CR: Predictors of adoption and maintenance of vigorous physical activity in men and women. Prev Med 1992, 21:237-251.

20. Gebel K, Bauman A, Owen N: Correlates of non-concordance between perceived and objective measures of walkability. Ann Behav Med 2009, 37:228-238.

21. Humpel N, Owen N, Leslie E: Environmental factors associated with adults' participation in physical activity: a review. Am J Prev Med 2002, 22:188-199.

22. McGinn AP, Evenson KR, Herring AH, Huston SL, Rodriguez DA: Exploring associations between physical activity and perceived and objective measures of the built environment. J Urban Health 2007, 84:162-184

23. Evenson KR, Scott MM, Cohen DA, Voorhees CC: Girls' perception of neighborhood factors on physical activity, sedentary behavior, and BMI. Obesity (Silver Spring) 2007, 15:430-445.

24. Foster S, Giles-Corti B: The built environment, neighborhood crime and constrained physical activity: an exploration of inconsistent findings. Prev Med 2008, 47:241-251.

25. Troped PJ, Saunders RP, Pate RR, Reininger B, Ureda JR, Thompson SJ: Associations between self-reported and objective physical environmental factors and use of a community rail-trail. Prev Med 2001, 32:191-200

26. Velasquez KS, Holahan CK, You X: Relationship of perceived environmental characteristics to leisure-time physical activity and meeting recommendations for physical activity in Texas. Prev Chronic Dis 2009, 6:A24.

27. Ball K, Jeffery RW, Crawford DA, Roberts RJ, Salmon J, Timperio AF: Mismatch between perceived and objective measures of physical activity environments. Prev Med 2008, 47:294-298.

28. Lee RE, Mama SK, Medina AV, Reese-Smith JY, Banda JA, Layne CS, Baxter M, O'Connor DP, McNeill L, Estabrooks PA: Multiple Measures of Physical Activity, Dietary Habits and Weight Status in African American and Hispanic or Latina Women. J Community Health 2011, PMID: 21519867.

29. Maternal and Infant Health Assessment, California Department of Health Services, Maternal, Child and Adolescent Health/Office of Family Planning Branch, 1999-2004. [http://www.cdph.ca.gov/data/surveys/Pages/ MaternalandInfantHealthAssessment(MIHA)survey.aspx].

30. Sarnoff $R$, Hughes $D$ : Increasing health insurance coverage in the first year of life. Matern Child Health J 2005, 9:343-350.

31. Sjostrom M, Bull F, Craig C: Towards standardized global assessment of health-related physical activity the International Physical Activity Questionnaire (IPAQ). Med Sci Sports Exerc 2002, 33S.

32. Layne CS, Mama SK, Banda JA, Lee RE: Development of an ecologically valid approach to assess moderate physical activity using accelerometry in community dwelling women of color: A cross-sectional study. Int $J$ Behav Nutr Phys Act 2011, 8:1-7.

33. Thomas S, Reading J, Shepherd RJ: Revision of the physical activity readiness questionnaire (PAR-Q). Can J Sport Sci 1992, 17:338-345.

34. McAlexander KM, Mama SK, Medina A, O'Connor DP, Lee RE: Concordance and correlates of direct and indirect built environment measurement among minority women. Am J Health Promot 2011. 
35. Parmenter B, McMillan T, Cubbin C, Lee R: Developing Geospatial Data Management, Recruitment, and Analysis Techniques for Physical Activity Research. Journal of the Urban and Regional Information Systems Association 2008, 20:13-19.

36. Diez-Roux AV: Bringing context back into epidemiology: variables and fallacies in multilevel analysis. Am J Public Health 1998, 88:216-222.

37. Lee RE, Booth KM, Reese-Smith JY, Regan G, Howard HH: The Physical Activity Resource Assessment (PARA) instrument: evaluating features, amenities and incivilities of physical activity resources in urban neighborhoods. Int J Behav Nutr Phys Act 2005, 2:13.

38. Heinrich KM, Lee RE, Regan GR, Reese-Smith JY, Howard HH, Haddock CK, Poston WS, Ahluwalia JS: How does the built environment relate to body mass index and obesity prevalence among public housing residents? Am J Health Promot 2008, 22:187-194.

39. McAlexander KM, Banda JA, McAlexander JW, Lee RE: Physical Activity Resource Attributes and Obesity in Low-Income African Americans. J Urban Health 2009, 86:696-707.

40. Clifton K, Levi Smith A, Rodriguez D: The development and testing of an audit for the pedestrian environment. Land Urb Plan 2007, 80:95-110.

41. United States Department of Health and Human Services: The 2007 HHS Poverty Guidelines. [http://aspe.hhs.gov/POVERTY/07poverty.shtml].

42. Hoehner CM, Brennan Ramirez LK, Elliott MB, Handy SL, Brownson RC: Perceived and objective environmental measures and physical activity among urban adults. Am J Prev Med 2005, 28:105-116.

43. Center for Disease Control and Prevention: Physical Activity. [http://www. cdc.gov/physicalactivity/index.html].

44. Lackey KJ, Kaczynski AT: Correspondence of perceived vs. objective proximity to parks and their relationship to park-based physical activity. Int J Behav Nutr Phys Act 2009, 6:53.

45. McCormack GR, Spence JC, Berry T, Doyle-Baker PK: Does perceived behavioral control mediate the association between perceptions of neighborhood walkability and moderate- and vigorous-intensity leisuretime physical activity? J Phys Act Health 2009, 6:657-666.

doi:10.1186/1479-5868-8-72

Cite this article as: McAlexander et al:: The concordance of directly and indirectly measured built environment attributes and physical activity adoption. International Journal of Behavioral Nutrition and Physical Activity 2011 8:72.

\section{Submit your next manuscript to BioMed Central and take full advantage of:}

- Convenient online submission

- Thorough peer review

- No space constraints or color figure charges

- Immediate publication on acceptance

- Inclusion in PubMed, CAS, Scopus and Google Scholar

- Research which is freely available for redistribution 